\title{
Thermal conductivity reduction in core-shell nanowires
}

\author{
Ming $\mathrm{Hu},{ }^{1, *}$ Xiaoliang Zhang, ${ }^{1,2}$ Konstantinos P. Giapis, ${ }^{3, \dagger}$ and Dimos Poulikakos ${ }^{1, \ddagger}$ \\ ${ }^{1}$ Laboratory of Thermodynamics in Emerging Technologies, Department of Mechanical and Process Engineering, \\ ETH Zurich, 8092 Zurich, Switzerland \\ ${ }^{2}$ Center for Heat and Mass Transfer, Institute of Engineering Thermophysics, Chinese Academy of Sciences, Beijing 100190, China \\ ${ }^{3}$ Division of Chemistry and Chemical Engineering, California Institute of Technology, Pasadena, California 91125, USA
}

(Received 27 April 2011; revised manuscript received 12 August 2011; published 29 August 2011)

\begin{abstract}
Nanostructuring of thermoelectric materials bears promise for manipulating physical parameters to improve the energy conversion efficiency of thermoelectrics. Using nonequilibrium molecular dynamics, we investigate how the thermal conductivity can be altered in core-shell nanocomposites of $\mathrm{Si}$ and $\mathrm{Ge}$. By calculating the phonon vibrational density of states and performing normal mode analysis, we show that the thermal conductivity decreases when phonon-transport becomes diffusion-dominated and unveil a competition between modes from the various regions of the nanocomposite (core, interface, and shell). The effects of nanowire length, cross section, and temperature on thermal conductivity are explicitly considered. Surprisingly, the thermal conductivity variation with nanowire length is much weaker than in pure nanowires. Also, the thermal conductivity is almost independent of temperature in the wide region between 50 and $600 \mathrm{~K}$, a direct result of confinement of the core by the shell. These results suggest that core-shell nanowires are promising structures for thermoelectrics.
\end{abstract}

DOI: 10.1103/PhysRevB.84.085442

PACS number(s): 63.22.Gh, 65.80.-g, 63.20.D-, 66.70.-f

\section{INTRODUCTION}

Thermoelectric materials convert temperature gradients to electric current with no moving parts. Thus, they could improve energy utilization by reducing heat wasted as part of thermodynamic inefficiencies. The energy conversion efficiency of thermoelectric devices is characterized by the dimensionless coefficient: $Z T=S^{2} \sigma T / \kappa$, where $S, \sigma$, and $\kappa$, are the Seebeck coefficient, electrical conductivity, and thermal conductivity of the material, respectively, and $T$ is the absolute temperature. $Z T$ values in the 3-4 range are needed for thermoelectrics to find widespread applications. Unfortunately, even improved bulk materials seldom have a $Z T \geqslant 1$. Increasing $Z T$ has proven to be challenging due to the strong interdependence of the physical parameters involved in electronic and phononic transport. ${ }^{1}$ As an alternative to designing new bulk materials with larger $Z T$ values, nanostructuring of existing thermoelectrics has emerged as a promising way to improve thermoelectric performance by manipulating phononic transport. ${ }^{2}$

Indeed, the thermal conductivity of relatively short $(<300 \mathrm{~nm})$ Si nanowires (NWs) fabricated from suspended thin Si films has been shown experimentally to be significantly lower than that of bulk Si, which was attributed to increased phonon scattering at the confining walls of the nanowires. ${ }^{3,4}$ The origin of the high thermoelectric performance in Si NWs was discussed in Chen et al. ${ }^{5}$ Additional methods to reduce the thermal conductivity were proposed in the literature, some supported by predictions from atomistic simulations. For example, creating an amorphous layer ${ }^{6}$ on the surface of Si NWs was calculated to reduce the thermal conductivity by two orders of magnitude in comparison to bulk silicon, something that could be accomplished by heating the Si NW up to a temperature very close to its melting point followed by fast quenching. In another example, creating a nanotube by conceptually drilling a small hole in the center of Si NWs was calculated to also reduce the thermal conductivity due to increased phonon scattering. ${ }^{7}$ Recently, Si/Ge core-shell nanocomposites ${ }^{8,9}$ were shown to significantly reduce the thermal conductivity through phonon scattering at the interface, while offering a simpler structure to pursue experimentally. Using molecular dynamics (MD) simulations, ${ }^{8}$ it was shown that a Ge layer of only 1 to 2 unit cell (uc) thickness conformal on a Si nanowire can lead to a $75 \%$ decrease in thermal conductivity at room temperature as compared to uncoated Si NWs. By analyzing the vibrational density of states and the participation ratio of each specific phonon mode, it was demonstrated that the reduction in the thermal conductivity of $\mathrm{Si} / \mathrm{Ge}$ core-shell nanowires originated in the depression and localization of both low-frequency phonon modes at the $\mathrm{Si} / \mathrm{Ge}$ interface, while high-frequency nonpropagating diffusive modes were also altered. ${ }^{8}$

In this paper, the concept of core-shell nanocomposites (Ref. 8) is systematically investigated with the aim to uncover the thermal conductivity dependence on important parameters, such as length, cross section, interfacial strength, and temperature. Confinement effects in the core-shell structures lead to a thermal conductivity behavior markedly different from that of pure nanowires. By reversing the core and shell materials, the ensuing $\mathrm{Ge}$-core/Si-shell structure was found to have an even lower thermal conductivity with exciting implications for thermoelectrics. The remainder of the paper is organized as follows. In Sec. II, we describe the model structures and the MD methodology used to determine the thermal conductivity of the nanowires. In Sec. III and Sec. IV, we present and discuss the results of nonequilibrium MD simulations on $\mathrm{Si}$-core/Ge-shell and Ge-core/Si-shell nanowires, respectively. Finally, the main points are summarized in Sec. V.

\section{MODEL STRUCTURES AND SIMULATION METHODOLOGY}

Our model system of Si-core/Ge-shell NW consists of a square cross section single-crystalline $\mathrm{Si}$ NW covered by a Ge layer. The NW extends in the [001] direction, i.e. the longitudinal direction, which is set along the $z$ axis. The surfaces in transverse directions ( $x$ and $y$ axes) for both $\mathrm{Si}$ 
and $\mathrm{Ge}$ are each (110) type. The cross section size, $(n \times n)$, in the $x$ and $y$ directions, varies from $n=2$ to 20, corresponding to a cross-sectional width from 0.77 to $7.7 \mathrm{~nm}$, respectively. In order to study the length dependence of thermal conductivity, for the case of $n=9$, the number of Si unit cells stacked up longitudinally varies from 25 to 2002, yielding a nanowire 13.6 to $1086 \mathrm{~nm}$ long. The corresponding number of Ge unit cells in the longitudinal direction changes from 24 to 1927, taking into consideration the [001] lattice mismatch between $\mathrm{Si}$ and $\mathrm{Ge}$. The structure of $\mathrm{Ge}$-core/Si-shell NWs was generated by reversing the unit cell number and lattice constant of the $\mathrm{Si}$ core and Ge shell in the longitudinal direction so that the same length is maintained as compared to the Si-core/Ge-shell NWs. Details on how to construct the initial structure can be found in Ref. 8.

The Stillinger-Weber (SW) potential was used to describe the interactions in the $\mathrm{Si}-\mathrm{Ge}$ system with diamond structure. This potential uses two-body and three-body terms to stabilize the diamond lattice. The original parameters were developed to provide an approximate description of condensed phases of $\mathrm{Si}^{10}$ Parameters for Ge were determined by fitting to experimental data. ${ }^{11}$ In the simulation studies described herein, the parameters of Refs. 10 and 11 were adopted to describe Si$\mathrm{Si}$ and $\mathrm{Ge}-\mathrm{Ge}$ interactions. Parameters for Si-Ge interactions were obtained by taking the arithmetic mean of $\mathrm{Si}$ and $\mathrm{Ge}$ parameters for $\sigma_{\mathrm{Si}-\mathrm{Ge}}$ and the geometric mean for $\lambda_{\mathrm{Si}-\mathrm{Ge}}$ and $\varepsilon_{\mathrm{Si}-\mathrm{Ge}}$, as suggested in the literature. ${ }^{12,13}$

All MD calculations were performed using the LAMMPS ${ }^{14}$ package with a time step of $1 \mathrm{fs}$ throughout. After equilibrating the system, we computed its thermal conductivity using nonequilibrium MD. In order to establish a temperature gradient along the longitudinal direction, the atoms close to the two ends of the NW (about 8 unit cells long) were placed into hot and cold baths with temperatures $T_{\mathrm{L}}$ and $T_{\mathrm{R}}$ for the left and right end, respectively. Nosé-Hoover thermostats ${ }^{15}$ were applied. The total heat flux in the longitudinal direction is defined as ${ }^{16}$

$$
\begin{gathered}
J_{L}(t)=\frac{1}{V}\left\{\sum_{i} v_{i, L} \varepsilon_{i}+\frac{1}{2} \sum_{i, j, i \neq j} \gamma_{i j, L}\left(\vec{f}_{i j} \cdot \vec{v}_{j}\right)\right. \\
\left.+\sum_{i, j, k} \gamma_{i j, L}\left[\vec{f}_{j}(i j k) \cdot \vec{v}_{j}\right]\right\} \\
\varepsilon_{i}=\frac{1}{2} m_{i} v_{i}^{2}+\frac{1}{2} \sum_{j} \phi_{2}\left(\gamma_{i j}\right)+\frac{1}{2} \varepsilon \sum_{j k} h\left(\gamma_{i j}, \gamma_{i k}, \theta_{j i k}\right), \\
\vec{f}_{j}(i j k)=-\varepsilon \nabla_{j} h_{3}\left(\gamma_{i j}, \gamma_{j k}, \theta_{j i k}\right),
\end{gathered}
$$

where the subscript $L$ denotes a quantity in the longitudinal direction, $\varepsilon$ is the energy scalar, and $h$ is the three-body interaction form in original SW potential, $v_{i}$ is the velocity of atom $i, \varepsilon_{i}$ is the local site energy, $\gamma_{i j}$ is the relative distance between atom $i$ and $j, \vec{f}_{i j}$ is the two-body force between atom $i$ and $j, \vec{f}_{j}(i j k)$ is the three-body interactions between atoms $i$, $j$, and $k, \phi_{2}$ and $h_{3}$ are the two-body and three-body potential energy, respectively, and $V$ is the volume of the region selected to calculate the heat flux. The thermal conductivity of the nanowire is calculated from

$$
\kappa=-\frac{\bar{J}_{L}}{\partial T / \partial z},
$$

where $\bar{J}_{L}$ is the averaged heat flux in the longitudinal direction and $\partial T / \partial z$ is the temperature gradient determined from a linear fitting of the time-averaged temperature profile along the nanowire. The thermal conductivity of only the core can also be calculated using Eq. (2), where the heat flux from Eq. (1) is evaluated by summing over all atoms $i$ in the $\mathrm{Si}$ core, since the heat flux can be defined for a single atom by determining the contribution of every atom to each term in Eq. (1). A typical equilibration run took about 6-10 ns. After equilibration, a temperature gradient was imposed on the system, and the simulation was run for about 10-30 ns to establish steady heat flow. After that, the statistics for averaging the temperature profile and heat flux were sampled by running the simulation for 15-30 ns, depending on system size and its thermal conductivity. For nanowires with cross sections less than $2 \times 2 \mathrm{~nm}^{2}$, very long runs ( $\left.\sim 60 \mathrm{~ns}\right)$ are needed to obtain a longer time average with better statistics. Generally, the shorter the NW and the lower its thermal conductivity, the longer the run required for the system to reach steady state after imposing a temperature gradient. The simulation time must exceed a characteristic time dependent on wire length and thermal diffusivity, as follows

$$
\tau=\frac{L_{z}^{2}}{D_{T}}=\frac{L_{z}^{2}}{\kappa /\left(\rho C_{p}\right)},
$$

where $L_{z}$ is the wire length, $D_{T}$ the thermal diffusivity, $\kappa$ the thermal conductivity, $\rho$ the density, and $C_{p}$ the specific heat capacity, respectively. For a typical wire length of $164 \mathrm{~nm}$, $\kappa \sim 5 \mathrm{~W} / \mathrm{mK}, \rho=2.33 \times 10^{3} \mathrm{~kg} / \mathrm{m}^{3}, C_{p} \sim 530 \mathrm{~J} / \mathrm{kg} / \mathrm{K}$ for thin nanowire, ${ }^{17}$ we obtain $\tau \sim 5.6 \mathrm{~ns}$. Thus the $10-30 \mathrm{~ns}$ simulation time is sufficient for the system to establish steady heat flow.

\section{RESULTS OF SI-CORE/GE-SHELL NANOWIRES}

In this section, we present simulation results on the dependence of the thermal conductivity of $\mathrm{Si}$-core/Ge-shell nanowires on length, temperature, cross section, and Si-Ge interfacial structure. The results are discussed in terms of the vibrational density of states (VDOS) for selected nanowire lengths accompanied by normal mode analysis.

\section{A. Length dependence}

The calculated thermal conductivity of a pure Si NW (cross section of $9 \times 9$ unit cells), plotted in Fig. 1(a), is found to increase rapidly with nanowire length below $\sim 300 \mathrm{~nm}$. This behavior is in agreement with the work of Wang et al. ${ }^{18}$ Thereafter, the thermal conductivity varies more slowly approaching asymptotically a constant value at lengths over $1200 \mathrm{~nm}$. The slow increase above $300 \mathrm{~nm}$ implies that the NW is in the diffusive phonon transport regime. Further analysis showed that the reciprocal of the thermal conductivity of a pure Si NW varies linearly with the reciprocal of the 
nanowire length in the entire length range studied, as shown in Fig. 1(b). Linear fitting of the datapoints using the function ${ }^{16}$

$$
\frac{1}{\kappa}=\frac{1}{\kappa_{\infty}^{N W}}\left(\frac{l_{N W}}{L_{z}}+1\right),
$$

where $\kappa_{\infty}^{N W}$ is the thermal conductivity of an infinitely long $\mathrm{NW}$ and $l_{N W}$ is the effective phonon mean free path in the $\mathrm{NW}$, yields $\kappa_{\infty}^{N W}=22.4 \mathrm{~W} / \mathrm{mK}$ and $l_{N W}=79.2 \mathrm{~nm}$. Note that both $\kappa_{\infty}^{N W}$ and $l_{N W}$ are functions of the NW diameter and temperature. Hence, the obtained values only apply to the case of cross section of $9 \times 9$ unit cells, i.e. $3.5 \times 3.5 \mathrm{~nm}^{2}$ with (110) surface structure and temperature of $300 \mathrm{~K}$. Since our cross section and surface orientation of the pure Si NW is different from previous studies, ${ }^{18-20}$ no exact comparison can be made. For consistency with previous studies, we run one more case of pure Si NW with an exact cross section of $4 \times$ $4 \times 302$ unit cells and (100) surface structure. We obtained a thermal conductivity of $4.39 \pm 0.50 \mathrm{~W} / \mathrm{mK}$, which is in good agreement with the value of $\sim 4 \mathrm{~W} / \mathrm{mK}$ (read from graph) reported in literature. ${ }^{19}$ Nevertheless, our estimated $\kappa_{\infty}^{N W}$ caps all the data reported in Refs. 18 and 19, where a thinner $\mathrm{Si}$ $\mathrm{NW}$ with (100) surface was used, and $l_{N W}$ is very close to the value of 35.9-74.4 nm estimated for thinner Si NW. ${ }^{19}$

The thermal conductivity of Si-core/Ge-shell NW coated with 2 unit cells of Ge is also plotted in Fig. 1 as a function of wire length. We first notice the larger thermal conductivity of the Si core compared to the overall thermal conductivity of the core-shell nanowire. This can be explained by writing the overall thermal conductivity $\left(\kappa_{\text {tot }}\right)$ as the cross sectional area weighted average of the core $\left(\kappa_{\text {core }}\right)$ and shell $\left(\kappa_{\text {shell }}\right)$ conductivities

$$
\kappa_{\mathrm{tot}}=\kappa_{\mathrm{core}} \times A_{\mathrm{core}} / A_{\mathrm{tot}}+\kappa_{\text {shell }} \times A_{\text {shell }} / A_{\mathrm{tot}},
$$

where $A$ is cross-sectional area with $A_{\text {tot }}=A_{\text {core }}+A_{\text {shell }}$. Due to the strong phonon interference at the core-shell interface, we expect that $\kappa_{\text {shell }}<\kappa_{\text {core }}$ when the shell is very thin-in this case, $\kappa_{\text {tot }}$ is slightly lower than $\kappa_{\text {core }}$. Note that sometimes $\kappa_{\text {shell }}>\kappa_{\text {core }}$, in that case, $\kappa_{\text {tot }}$ could be larger than $\kappa_{\text {core }}$ (see below). In comparison to the pure $\mathrm{Si} \mathrm{NW}$, there is no significant increase in the thermal conductivity of the core-shell NW over the entire length range studied [Fig. 1(a)], i.e. the length dependence is much weaker. To this end, the conductivity increases by only $\sim 12 \%$ when the wire length increases from 300 to $1,086 \mathrm{~nm}$. We interpret these results to mean that the core-shell NW is in the ballistic-transport regime of heat conduction for $L_{z} \leqslant 100 \mathrm{~nm}$ and in the diffusion-transport regime for $L_{z}>300 \mathrm{~nm}$, since the phonon mean free path of $\mathrm{Si}$ core with Ge shell is obtained from Eq. (4) and Fig. 1(b) to be $71.4 \mathrm{~nm}$. The same behavior is found for the Si core without the shell. Extrapolation of $\frac{1}{\kappa}$ vs $\frac{1}{L_{z}}$ for infinitely long core-shell nanowires in Fig. 1(b) yields a thermal conductivity of the $\mathrm{Si}$ core of $6.51 \mathrm{~W} / \mathrm{mK}$, resulting in a reduction of $71 \%$ compared to that of an infinitely long pure $\mathrm{SiNW}$ with the same cross section [Fig. 1(a)]. Thus, the significant reduction in the thermal conductivity found for shorter core-shell nanowires holds true also for much longer ones, in sharp contrast to the case of pure Si NW.
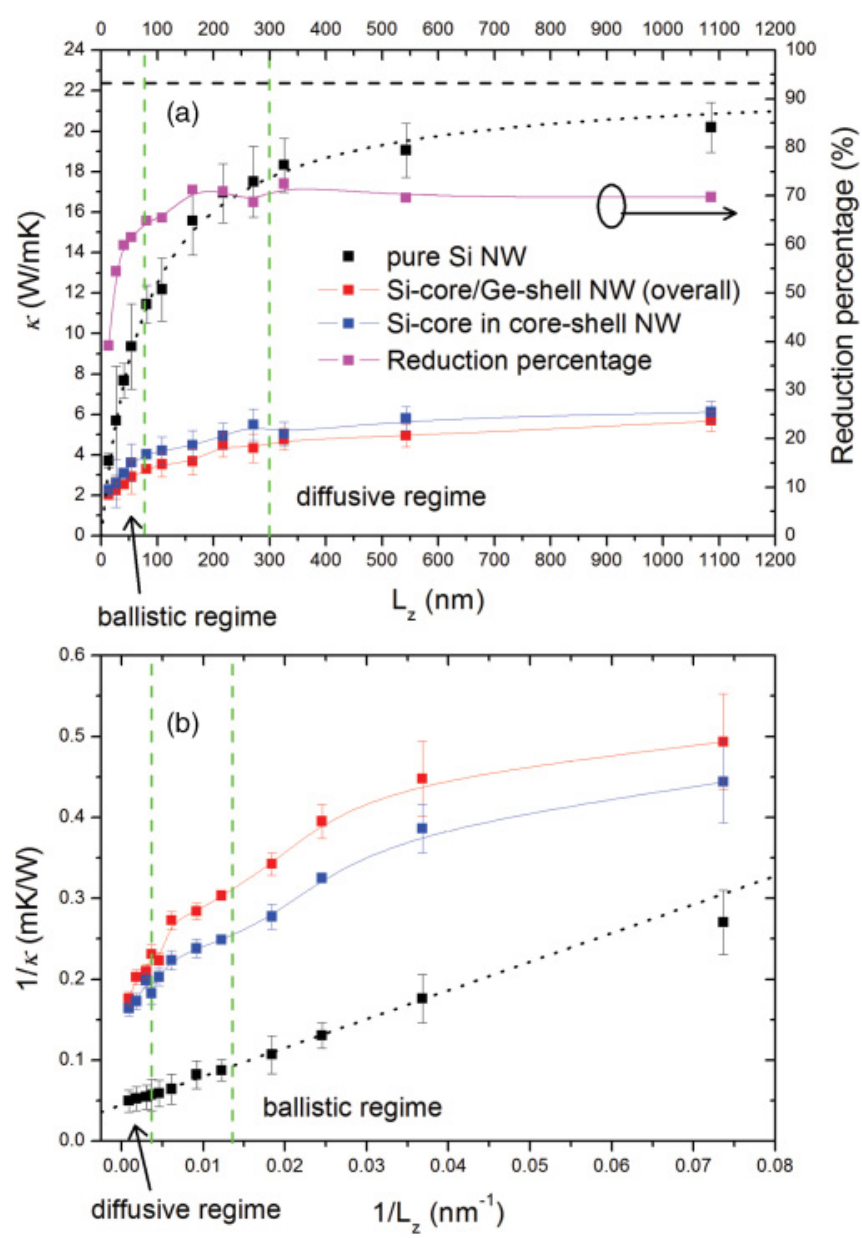

FIG. 1. (Color online) (a) Calculated thermal conductivity for a Si-core/Ge-shell structure vs nanowire length at $300 \mathrm{~K}$ with comparison to that of a pure Si nanowire. (b) Relationship between the reciprocal of thermal conductivity and the reciprocal of length. The dotted line represents a fitting to the MD results, while the black dashed line in panel (a) denotes the upper limit of the thermal conductivity of an infinitely long NW with the same cross section. The green dashed lines mark the suggested ballistic and diffusive regimes. The right axis in panel (a) shows the percent reduction in thermal conductivity of the $\mathrm{Si}$ core relative to that of a pure $\mathrm{Si}$ nanowire.

\section{B. Local vibrational density of states}

The reduction in thermal conductivity of the $\mathrm{Si}$-core/Geshell NW is attributed to the depression in the vibrational density of states (VDOS) of Si atoms on the nanowire surface upon addition of the Ge shell. ${ }^{8}$ The VDOS at the surface of pure $\mathrm{Si} \mathrm{NW}$ and those at the $\mathrm{Si} / \mathrm{Ge}$ interface are calculated by a Fourier transform of the atomic velocity autocorrelation function. Figure 2 compares the calculated VDOS for selected nanowire lengths. Addition of the Ge shell results in a large attenuation of the low-frequency modes, especially below $6 \mathrm{THz}$, which is caused by phonon interference at the $\mathrm{Si} / \mathrm{Ge}$ interface due to the lattice mismatch and the atomic mass difference between $\mathrm{Si}$ and Ge. Since low-frequency modes dominate heat transfer in $\mathrm{Si}$, their attenuation impacts adversely the thermal conductivity. As expected from bulklike behavior away from the surface, the VDOS of Si atoms at the 


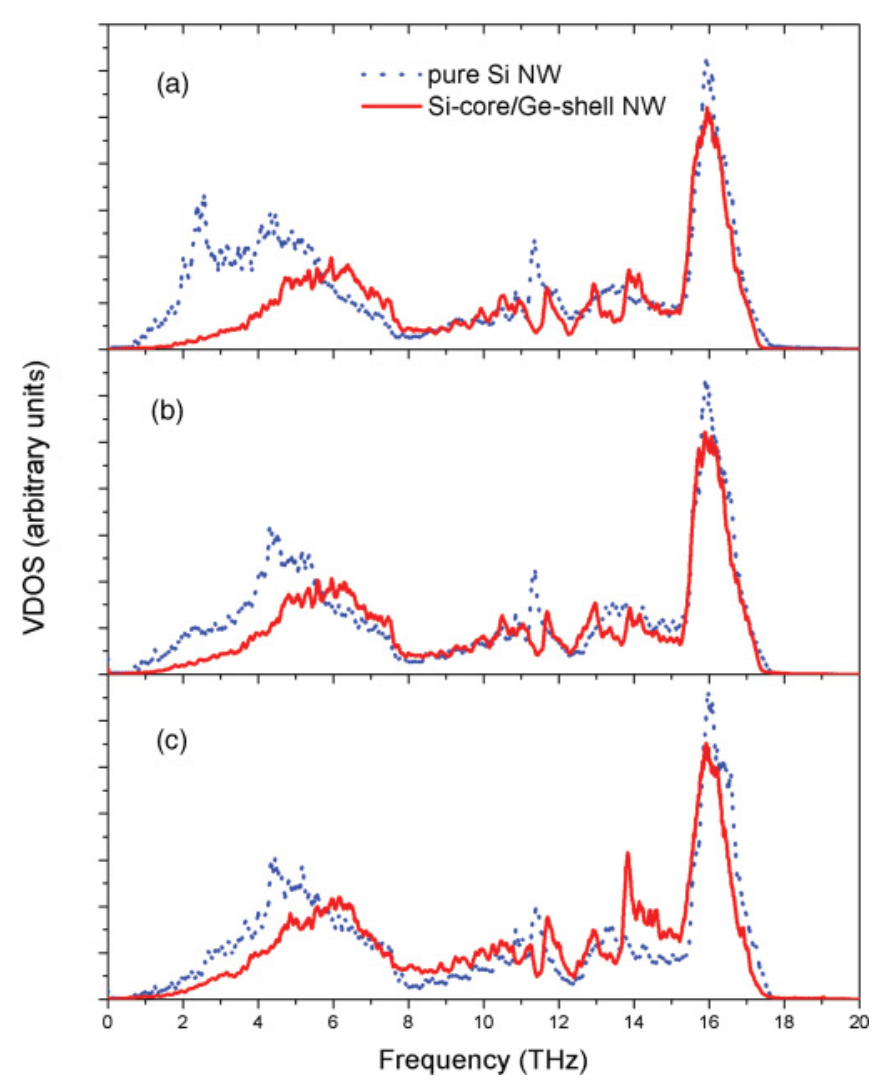

FIG. 2. (Color online) Comparison of vibrational density of states of $\mathrm{Si}$ atoms located on the nanowire surface in the absence and presence of a Ge shell of 2-unit-cell thickness. The core $\mathrm{Si}$ is a matrix of (a) $9 \times 9 \times 52$ u.c.; (b) $9 \times 9 \times 502$ u.c.; (c) $9 \times 9 \times$ 2002 u.c.

center of the Si core does not change much with added Ge layers (not shown for brevity).

\section{Temperature dependence}

The calculated temperature dependence of the thermal conductivity is reported in Fig. 3. Due to the quantum nature of thermal vibrations in the low-temperature range, classical molecular dynamics below $50 \mathrm{~K}$ are not reliable. The thermal conductivity of a pure $\mathrm{Si} \mathrm{NW}$ exhibits a sharp peak at low temperatures then decays rapidly as the temperature increases to $400 \mathrm{~K}$. Thereafter, the conductivity continues to decrease, but at a slower pace. The trend is identical to that of bulk $\mathrm{Si}^{21}$ and has also been confirmed experimentally for Si nanowires. ${ }^{3,22,23}$ The origin of this trend lies in the reduced dimensionality of the nanowire-a linear temperature dependence is expected for a quasi-one-dimensional system, indicative of ballistic thermal transport. A maximum occurs when phonon-phonon scattering starts playing a role (at $T \sim$ $50 \mathrm{~K}$ for bulk $\mathrm{Si}^{21}$ ) while anharmonic effects dominate in the high-temperature regime where $\kappa \sim 1 / T$.

Surprisingly, the thermal conductivity of core-shell nanowire remains constant throughout the entire temperature range studied, showing a perfect harmonic effect (Fig. 3). This temperature independence is believed to originate from the presence of nonpropagating vibrational modes similar to those contributing to heat transport in bulk amorphous

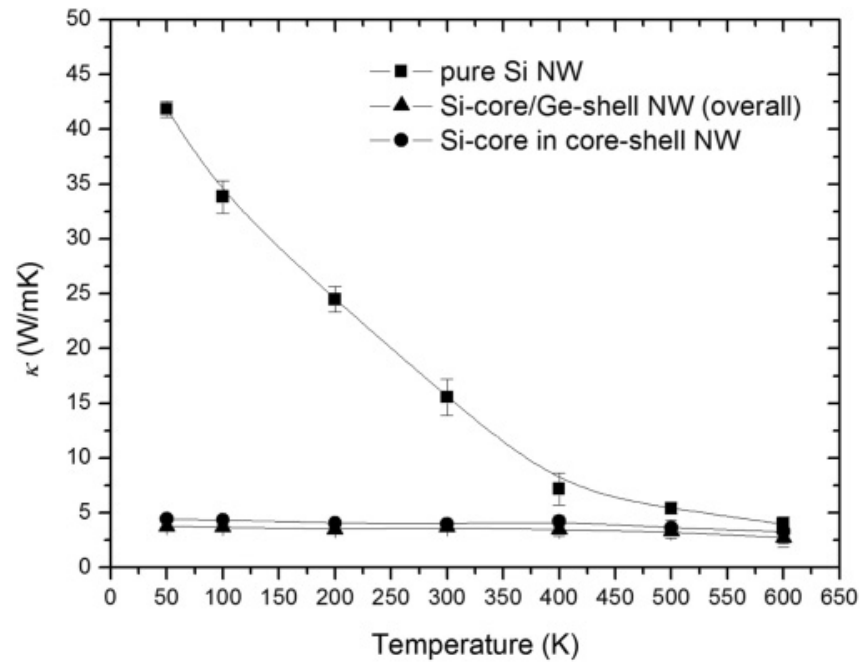

FIG. 3. Thermal conductivity of $9 \times 9 \times 302$ u.c. (164 nm long) nanowire as a function of temperature. The Ge layer on the core-shell structure is 2 unit cells thick.

Si and in thin Si NW with amorphized surface. ${ }^{24}$ Since the diffusivity of nonpropagating vibrational modes does not depend on temperature, the observed behavior suggests that the vibrational spectrum of the core-shell nanowire is dominated by diffusive modes. The temperature independence also suggests that $\mathrm{Si} / \mathrm{Ge}$ core-shell nanowires may have a considerably larger $Z T$ coefficient over pure Si nanowires at temperatures $\leqslant 300 \mathrm{~K}$.

\section{Cross-section dependence}

The nanowire cross section also influences thermal transport. As the lateral dimension is reduced to the nanometer scale, which is comparable to or even smaller than the phonon mean free path in a solid, phonon boundary scattering at the free surface of the nanowire becomes important in hindering phonon propagation in the solid. Thus, the influence of the core-shell boundary on thermal transport is expected to become more significant in thinner nanowires. Therefore, it is necessary to investigate how the thermal conductivity of a $\mathrm{Si}$-core/Ge-shell NW changes with thickness and compare it to that of a pure $\mathrm{Si}$ NW.

Simulation results for nanowires of varying square cross sections (or thickness, $D$ ) are reported in Fig. 4. Whereas the thermal conductivity of a pure $\mathrm{Si} \mathrm{NW}$ increases monotonically with thickness up to $8 \mathrm{~nm}$, the conductivity of Si-core/Geshell NW behaves in a nonmonotonic fashion. For very thin and short Si NWs $(D<1.2 \mathrm{~nm}, L=164 \mathrm{~nm})$, where the phonon transport is dominantly ballistic, the thermal conductivity is relatively independent of the thickness. In such cases, the thickness is much less than the phonon mean free-path, estimated to be 3.24-5.57 nm in a previous MD simulation. ${ }^{19}$ Consequently, the phonon boundary scattering at the free surface is dominantly specular. This independence for small diameter is consistent with the above discussion that ballistic phonon transport is dominant in the short length or small diameter regime. At the ballistic phonon transport limit, where all the populated phonons experience ballistic transport, the thermal conductance, $G=\kappa A / L$, is proportional 


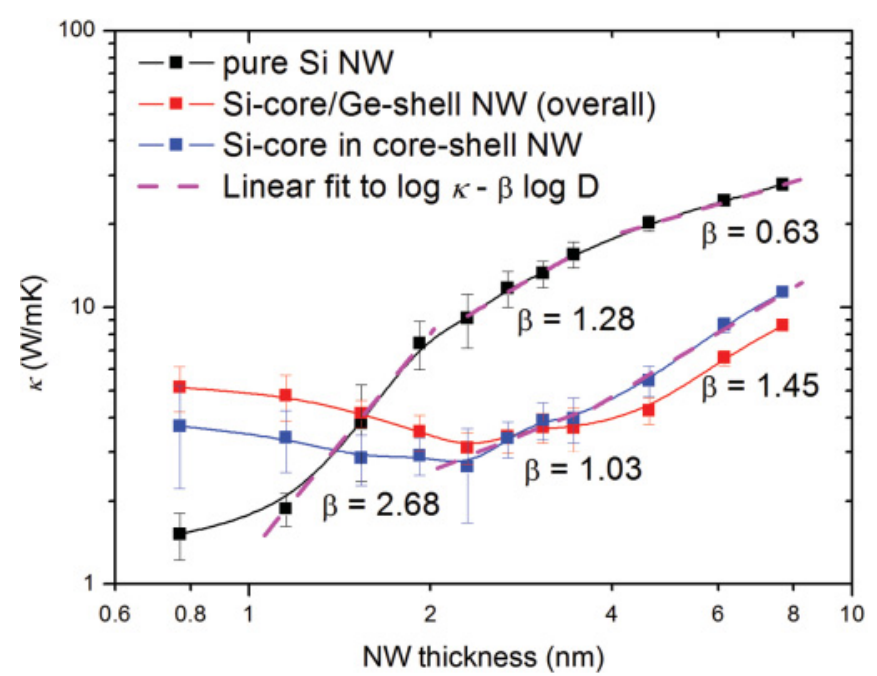

FIG. 4. (Color online) Calculated thermal conductivity of a square cross section $\mathrm{Si} / \mathrm{Ge}$ core-shell nanowire as a function of thickness at $300 \mathrm{~K}$. Results are also shown for a pure Si nanowire. All nanowires are $164 \mathrm{~nm}$ long and the Ge layer is 2 unit cells thick. The dashed lines are linear fits to the $\log \kappa \sim \beta \log D$ relationship with exponent $\beta$ indicated.

to the number of atoms per unit length, i.e. density $\times$ cross-sectional area. This means that, with constant density and the current definition of cross-sectional area $A=D^{2}$, the thermal conductivity $(\kappa=G L / A)$ is independent of the diameter.

It can be also seen from Fig. 4 that the thermal conductivity of pure $\mathrm{Si}$ NW increases dramatically with thickness. The slope of $\log \kappa-\beta \log D$ gradually decreases as $D$ increases, since the phonon mean free path gradually becomes shorter relative to $D$, i.e. diffusive phonon transport is gradually enhanced compared to ballistic phonon transport. The monotonic increase in the thermal conductivity of pure Si NWs and the decaying growth rate with diameter is consistent with previous theoretical work on other semiconductor nanowires based on the Boltzmann transport equation. ${ }^{25}$ Due to the large computational time requirements, we cannot run simulations for 164-nm-long and more than 10-nm-thick nanowires with (110)-type surfaces. However, the present results suggest that the thermal conductivity of a pure Si NW with infinite diameter will converge to a finite value. It should be emphasized that this value is not the conductivity of bulk $\mathrm{Si}$, since we are simulating a finite length of a Si NW, which contains the finite size effect in the heat flow direction and is different from the cases studied in Ref. 25.

Interestingly, the thermal conductivity of both the $\mathrm{Si} / \mathrm{Ge}$ core-shell nanowire as well as the $\mathrm{Si}$ core (taken alone) vs thickness shows a markedly different behavior from that of a freestanding Si nanowire. Initially, both conductivities are independent of thickness for $D<1.2 \mathrm{~nm}$. Then they slowly decrease to a minimum for a thickness of $\sim 2.3 \mathrm{~nm}$. After a transition region, the conductivity increases sharply with thickness, with an exponent $\beta$ much larger than that for the freestanding Si NW. We also found that the core-shell NW enters a fully diffusive regime at a much larger diameter than the freestanding Si NW. This behavior is a result of the

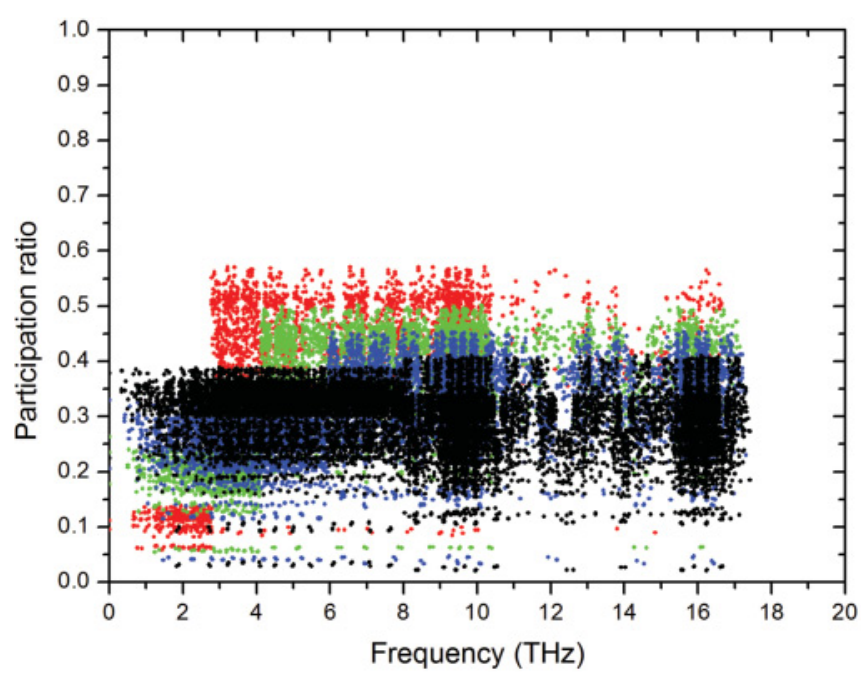

FIG. 5. (Color online) Participation ratio of each vibrational eigenmode for $\mathrm{Si} / \mathrm{Ge}$ core-shell nanowires with different core thickness. Red: $D=0.77 \mathrm{~nm}$; green: $1.54 \mathrm{~nm}$; blue: $2.31 \mathrm{~nm}$; and black: $3.46 \mathrm{~nm}$. In all cases, the Ge layer is 2 unit cells thick.

strong interference at the core-shell interface, which limits thermal transport to a smaller effective diameter. Furthermore, by comparing the thermal conductivity of the core-shell NW to its Si core, a crossover behavior becomes apparent. For thin nanowires $(D<2.3 \mathrm{~nm})$ the core-shell composite has higher thermal conductivity than the Si core NW, but for thicker nanowires $(D>4 \mathrm{~nm})$ the relative magnitudes switch.

To fully understand the minima and the crossover regions in Fig. 4, we performed normal mode analysis of vibrational properties of core-shell nanowires. First, we analyzed the mode localization by calculating the participation ratio $p_{\lambda}$, defined for each mode $\lambda$ as $^{26}$

$$
p_{\lambda}^{-1}=N \sum_{i}\left(\sum_{\alpha} \varepsilon_{i \alpha, \lambda}^{*} \varepsilon_{i \alpha, \lambda}\right)^{2},
$$

where $i$ sums over all the atoms studied, $\alpha$ is a Cartesian direction and sums over $x, y, z$, and $\varepsilon_{i \alpha, \lambda}$ the vibrational eigenvector component corresponding to the $\lambda$ th normal mode, and $N$ the total number of atoms. The participation ratio describes the fraction of atoms participating in a particular mode and, hence, it varies between $O(1)$ for delocalized states to $O(1 / N)$ for localized states. We selected a segment of $n \times$ $n \times 6 \mathrm{Si}$ covered by 2 unit cells of Ge NW, where $n$ ranges from 2 (corresponding to $D=0.77 \mathrm{~nm})$ to $9(D=3.46 \mathrm{~nm})$, and mimic the long core-shell NW by using periodic boundary conditions in the longitudinal direction.

Figure 5 compares the participation ratios of all modes in the spectrum for the $\mathrm{Si}$-core/Ge-shell NWs with different diameters of Si cores. In all cases, the Ge shell is 2 unit cells thick. There are several distinct features in the participation ratios. First, for small diameters $(D<2.31 \mathrm{~nm})$, there is a noticeable step between low-frequency $(\omega<3-4 \mathrm{THz})$ and intermediate-frequency modes $(3-4 \mathrm{THz}<\omega<10 \mathrm{THz})$, which hints to a nonuniform distribution of the participation ratios in the frequency spectrum. As we will see later, this stepwise change is caused by the competition between Si core 
and Ge shell. For very small Si core diameters, low-frequency modes are highly localized to those atoms on the surface of the Ge shell. The vibrations of atoms in this case are dominated by the Ge shell surface, and consequently their participation ratios are quite low. Therefore, the main contribution to the overall thermal conductivity of the core-shell NW is by the Ge shell, which is slightly larger than that of the Si core due to its larger cross-sectional area. As the diameter increases, the stepwise change shifts to intermediate frequencies, which implies that more low-frequency modes are localized. Simultaneously, the participation ratio is increased in the low-frequency range, while it is reduced in the high-frequency range $(\omega>10 \mathrm{THz})$, until it becomes uniform throughout the entire vibrational frequency range at $D=3.46 \mathrm{~nm}$. For moderate diameters $(2.31<D<3.46 \mathrm{~nm})$, the $\mathrm{Si}$ core-atom contributions begin to surpass those of the Ge shell, which is consistent with the increased cross-sectional area of the $\mathrm{Si}$ core and the reduced phonon scattering. Finally, for larger diameters $(D \geqslant 3.46 \mathrm{~nm})$, a larger number of $\mathrm{Si}$ atoms in the core participate in the low-frequency modes, and the vibrations in the NW gradually become $\mathrm{Si}$ dominated. The participation ratio is almost uniform in the range of frequencies below $10 \mathrm{THz}$, while it is highly concentrated around $16 \mathrm{THz}$ with some localized modes in between. Note that 10 and $16 \mathrm{THz}$ are the cutoff frequencies in the VDOS of Ge shell and Si core, respectively. This analysis suggests that the presence of the Ge shell induces the $\mathrm{Si}$ atoms in the core to vibrate resonantly with the $\mathrm{Ge}$ atoms, which constitutes direct evidence of a confinement effect of the Ge shell on the vibrational modes of the $\mathrm{Si}$ core.

Although the participation ratio covers the spatial extension over the entire core-shell NW, it does not provide information on the relative contributions from individual modes from the three regions composing the entire $\mathrm{NW}$, i.e. the interior of $\mathrm{Si}$ core, $\mathrm{Si}-\mathrm{Ge}$ interfacial region, and $\mathrm{Ge}$ shell surface region. This regional contribution of modes can be characterized by the mode weight factor defined as ${ }^{27}$

$$
f_{j \prime, \lambda}=\sum_{j^{\prime}} \sum \alpha\left(\varepsilon_{j \alpha, \lambda}\right)^{2},
$$

where the prime denotes that sum over $j$ (atoms) and is alternatively restricted to the interior of the $\mathrm{Si}$ core, the $\mathrm{Si}-\mathrm{Ge}$ interface, and the Ge surface region. The Si-Ge interfacial region is defined by the Si and Ge atoms which have at least one neighbor from the other element within the cutoff distance of the force field. Consequently, the sum of the mode weight factors in the core-shell NW is equal to unity, i.e. $f_{\text {core }, \lambda}+f_{\text {interface, } \lambda}$ $+f_{\text {shell }, \lambda}=1$. As $f_{\text {shell }, \lambda} \rightarrow 1$ (or $f_{\text {core }, \lambda} \rightarrow 0$ ), the mode $\lambda$ tends to become more localized on the Ge shell surface and vice versa.

In Fig. 6, we plot the mode weight factors of the Si interior, the $\mathrm{Si}-\mathrm{Ge}$ interface, and the $\mathrm{Ge}$ surface for a $\mathrm{Si}$-core/Ge-shell $\mathrm{NW}$ as a function of the size of the Si core. At first glance, there are significant differences between the mode weight factors for the different regions with distinct steps between low-frequency and intermediate-frequency ranges. For very small Si cores $[D \leqslant 2.31 \mathrm{~nm}$, Figs. 6(a) and 6(b)], the low-frequency modes are dominated by the Ge shell. Thus, the $\mathrm{Ge}$ shell is mainly responsible for heat conduction in the

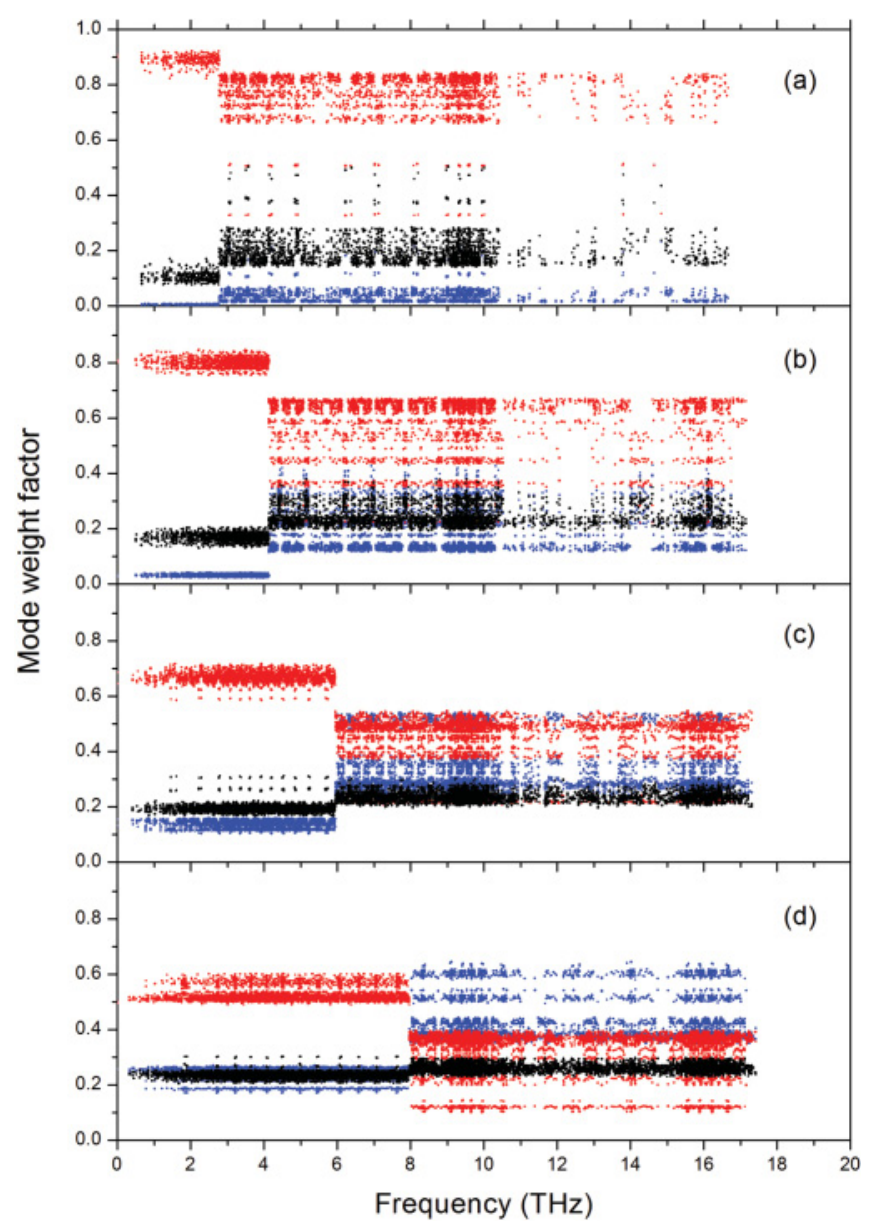

FIG. 6. (Color online) Fraction of each mode contained in Si core (blue dots), Si-Ge interface (black dots), and Ge shell (red dots) for different Si core thickness, (a) $0.77 \mathrm{~nm}$, (b) $1.54 \mathrm{~nm}$, (c) $2.31 \mathrm{~nm}$, and (d) $3.46 \mathrm{~nm}$. In all cases the Ge layer is 2 unit cells thick.

core-shell structure. This is consistent with the result of Fig. 4 that the overall thermal conductivity of Si-core/Ge-shell NW is higher than that of $\mathrm{Si}$ core alone. For the thinnest $\mathrm{Si}$ core [Fig. 6(a)], the mode weight factor of the Ge surface and $\mathrm{Si}$-Ge interface for low-frequency modes is around 0.9 and 0.1 , respectively, showing virtually no Si core atom participation. Increasing the $\mathrm{Si}$ core size allows more $\mathrm{Si}$ atoms to interfere with the vibrations of the Ge shell, causing a slight decrease in thermal conductivity, also seen in Fig. 4. For larger Si core sizes, the mode weight factors of the Ge shell and the $\mathrm{Si}$ core become comparable, while those of $\mathrm{Si}-\mathrm{Ge}$ interface remain almost unchanged [Fig. 6(c)]. This behavior implies that more atoms in the Si core participate in the vibrations of the core-shell structure and contribute to the overall heat transfer. This analysis is consistent with the minimum in thermal conductivity observed in Fig. 4. Further increase in the Si core size causes the mode weight factor of the Si core to exceed that of Ge shell [Fig. 6(d)], indicating that the vibrations in the core-shell NW are now dominated by the Si core. Thus, it is no longer surprising that the thermal conductivity of the Si core is higher than the overall thermal conductivity of the core-shell nanowire (Fig. 4). 


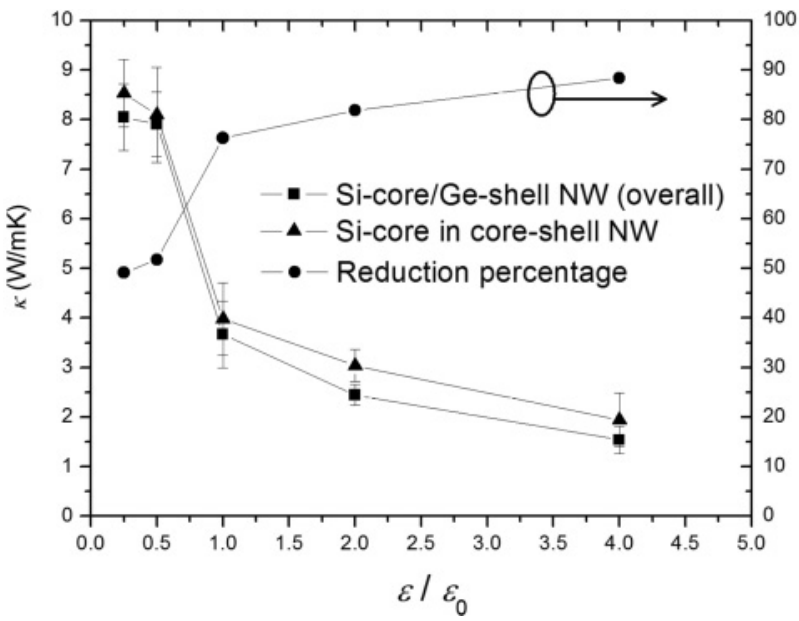

FIG. 7. Thermal conductivity of $9 \times 9 \times 302$ u.c. $(165 \mathrm{~nm}$ long) Si-core/Ge-shell NW as a function of the normalized interfacial strength. The right axis indicates the percent reduction in thermal conductivity of the Si core relative to that of a pure Si NW. The Ge layer is 2 unit cells thick.

\section{E. Effect of Si-Ge interfacial strength and structure}

The significant reduction in thermal conductivity of coreshell nanowires is a consequence of phonon interference at the Si-Ge interface, which in turn depends on the interfacial interaction strength. The latter dependence is clearly seen in Fig. 7. Here $\varepsilon_{0}$ denotes the original value obtained from the geometric mean of $\varepsilon_{\mathrm{Si}-\mathrm{Si}}$ and $\varepsilon_{\mathrm{Ge}-\mathrm{Ge}}$, which is the scaling factor of the interaction energy in the Stillinger-Weber potential. ${ }^{10,11}$ The thermal conductivity of both the core-shell NW and the Si core are found to drop considerably with interfacial interaction strength. This relationship can be understood in terms of vibrational coupling between modes in the Si core and Ge shell. When coupling is weak $\left(\varepsilon \leqslant \varepsilon_{0}\right)$, modes from the core and the shell contribute independently to the overall thermal conductivity. When the coupling is strong $\left(\varepsilon>\varepsilon_{0}\right)$, phonon interference impedes heat transfer. For example, if the interfacial strength can be made 4 times larger (by perhaps using a material other than $\mathrm{Ge}$ ), the thermal conductivity of the $\mathrm{Si}$ core will be reduced by $88.4 \%$ as compared to a pure $\mathrm{Si}$ $\mathrm{NW}$ of equal size. This result suggests that interference at the core-shell interface plays a dominant role in determining the thermal conductivity of Si core, while the thermal conductivity of the outer shell is less important.

In order to investigate the effect of the Si-Ge interfacial structure on the thermal transport properties of the core-shell $\mathrm{NW}$, we considered two additional types of Si-Ge interfaces

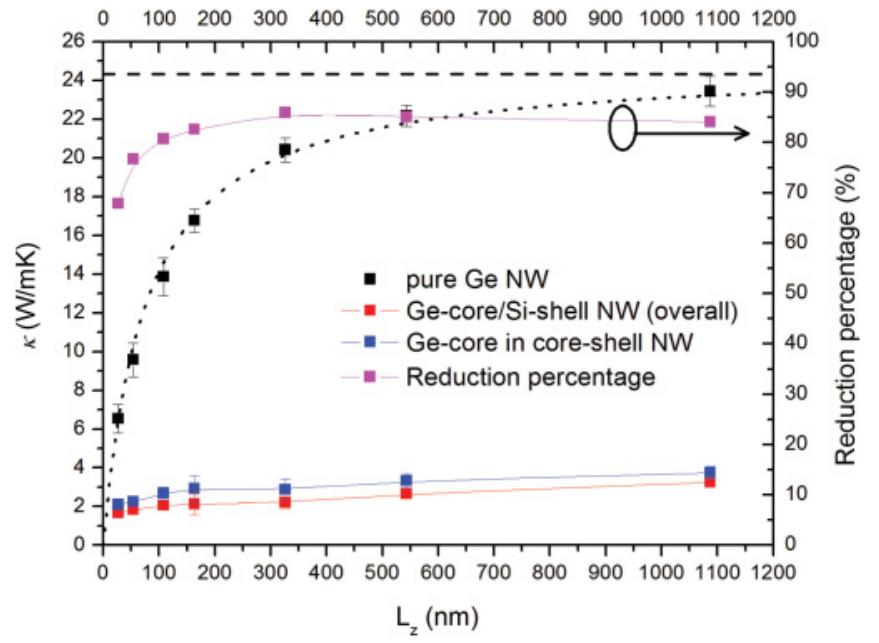

FIG. 8. (Color online) Calculated thermal conductivity for a Gecore/Si-shell structure vs nanowire length at $300 \mathrm{~K}$ with comparison to that of a pure Ge nanowire. The dotted line is fitting to the MD results, and the dashed line denotes the upper limit of the thermal conductivity of an infinitely long pure Ge NW with same cross section size. The right axis shows the percent reduction in thermal conductivity of the Ge core relative to that of pure Ge NW.

for the selected $9 \times 9 \times 302$ unit cell Si NW: one with 2 unit cells of Ge lattice-matched (epitaxial) to the $\mathrm{Si}$ surface, and the other with subsequent annealing treatment, as follows. The core-shell NW was heated to $1000 \mathrm{~K}$ in $1 \mathrm{~ns}$ and kept at this temperature for an additional $1 \mathrm{~ns}$. We note that the $\mathrm{Ge}$ layer did not melt during the relaxation at $1000 \mathrm{~K}$ because of superheating induced by the strong interfacial interaction with $\mathrm{Si}$ and the Stillinger-Weber potential parameter for $\mathrm{Ge}$, as we can see later in Sec. IV. Then the system was cooled down to room temperature with the same cooling rate, followed by room temperature relaxation before calculating the thermal conductivity. The results are compared in Table I. The epitaxially formed Si-Ge interface reduces significantly the overall thermal conductivity of the Si-Ge composite and its $\mathrm{Si}$ core, as compared to a pure Si nanowire. In this case, however, the nanowire was severely curved in the longitudinal direction due to the larger lattice constant of $\mathrm{Ge}$, leading to a large uncertainty in the calculated thermal conductivity of the core-shell NW. In contrast, annealing the core-shell NW yields further reduction (to $82.7 \%$ ) in the thermal conductivity of both the combined core-shell structure and the Si core alone. The larger reduction is the result of a much rougher and less uniform interface, which increases strongly the scattering of phonons propagating in the $\mathrm{Si}$ core.

TABLE I. Calculated thermal conductivity of Si/Ge core-shell nanowires with different interfacial structures at $300 \mathrm{~K}$. The thermal conductivity of the Si core is also tabulated for comparison. The system size is $9 \times 9 \times 302$ unit cells with a 2-unit-cell layer of Ge. The values in parenthesis are the percent reduction in the thermal conductivity from the value of a pure Si nanowire.

\begin{tabular}{lcr}
\hline \hline & Overall thermal conductivity of the Si-Ge core-shell nanowire & Thermal conductivity of the Si core \\
\hline Direct buildup & $3.669 \pm 0.668(76.4 \%)$ & $3.977 \pm 0.735(74.4 \%)$ \\
Epitaxial growth & $5.603 \pm 1.781(64.0 \%)$ & $6.132 \pm 1.850(60.6 \%)$ \\
Annealing after direct buildup & $1.616 \pm 0.250(89.6 \%)$ & $2.694 \pm 0.485(82.7 \%)$ \\
\hline \hline
\end{tabular}




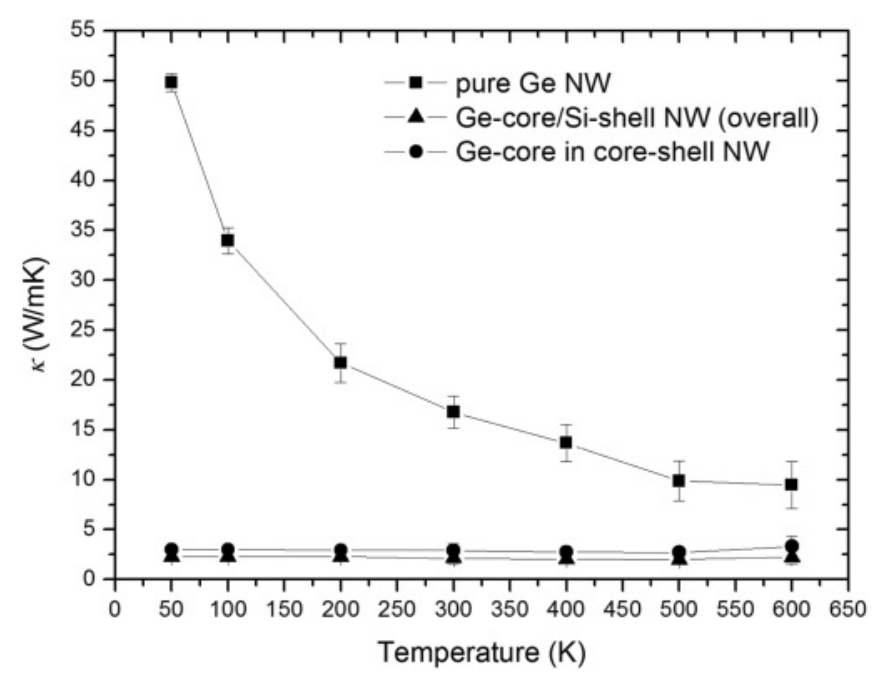

FIG. 9. Thermal conductivity of $9 \times 9 \times 292$ u.c. $(165 \mathrm{~nm}$ long $)$ $\mathrm{Ge}-\mathrm{core} / \mathrm{Si}$-shell $\mathrm{NW}$ as a function of temperature. For all the NWs, the $\mathrm{Si}$ layer is 2 unit cells thick.

\section{RESULTS FOR GE-CORE/SI-SHELL NANOWIRES}

It is instructive to study the thermal transport properties of the inverse nanocomposites, not in the least because $\mathrm{Ge}$-core/Si-shell nanowires have already been fabricated experimentally. ${ }^{28,29}$ Construction of Ge-core/Si-shell nanowires is straightforward by inverting the elements in the previous simulation. Figure 8 illustrates the length dependence of the thermal conductivity of Ge-core/Si-shell NWs. In all cases, a $9 \times 9$ Ge core was covered conformally by 2 unit cells of Si. Similar to pure Si NWs (Fig. 1), the thermal conductivity of the pure Ge NW initially increases sharply with length and then approaches asymptotically a constant value above $300 \mathrm{~nm}$. Fitting of the data using Eq. (4) yields $\kappa_{\infty}^{N W}=24.3 \mathrm{~W} / \mathrm{mK}$ and $l_{N W}=75.8 \mathrm{~nm}$. Note that, although the thermal conductivity of bulk Ge (experimental value $\sim 60 \mathrm{~W} / \mathrm{mK}$ ) is much lower than that of bulk $\mathrm{Si}$ $(\sim 150 \mathrm{~W} / \mathrm{mK})$, the calculated thermal conductivity of an infinitely long pure Ge NW is a bit larger than that of a pure Si NW with equal cross section. This is believed to be an artifact of the Stillinger-Weber potential parameter for Ge, which overestimates considerably the thermal conductivity and melting temperature of bulk Ge. ${ }^{30,31}$ Separate simulations showed that the Stillinger-Weber potential parameter for $\mathrm{Ge}$ proposed by Ding et al. ${ }^{11}$ yields a thermal conductivity of bulk Ge of $309.6 \pm 12.9 \mathrm{~W} / \mathrm{mK}$, which is several times larger than the experimental value. The thermal conductivity of the Ge-core/Si-shell NW does not change much for lengths between 27 and $1087 \mathrm{~nm}$. The behavior is identical for the Ge core taken alone. It is worth noting that a maximum reduction of $86.0 \%$ and $84.1 \%$ in the thermal conductivity of the Ge core was calculated for $L_{z}=327$ and $1087 \mathrm{~nm}$, respectively, as compared to the thermal conductivity of a freestanding Ge NW. The large reduction in thermal conductivity is attributed to the broader vibrational spectrum of the Si shell, which is expected to affect the entire range of vibrations of the $\mathrm{Ge}$ core, i.e. both low- and high-frequency modes. These results suggest that the Ge-core/Si-shell structure may be an even better candidate for higher $Z T$ values.
The temperature effect on the thermal conductivity of the Ge-core/Si-shell NWs was also studied and the results are plotted in Fig. 9. For a pure Ge NW, the thermal conductivity decays as $1 / T$ from 50 to $600 \mathrm{~K}$. In contrast, the thermal conductivity of the Ge-core/Si-shell NW remains almost constant, exhibiting a perfect harmonic effect throughout the entire temperature range. This trend and its explanation are identical to those described earlier for the inverse nanocomposite structure.

\section{CONCLUSIONS}

Comprehensive nonequilibrium molecular dynamics simulations of thermal transport in $\mathrm{Si} / \mathrm{Ge}$ and $\mathrm{Ge} / \mathrm{Si}$ core-shell nanowires have been performed to investigate whether such composites may offer advantages in thermoelectic applications. Specifically, the effects of the nanowire length, cross section, and temperature on the thermal conductivity of the nanocomposites were considered in detail. As compared to single-component nanowires, thermal transport in coreshell nanocomposites was found to differ considerably, as follows:

(i) Phonon transport in a core-shell nanowire becomes diffusion dominated at shorter lengths than in a pure nanowire and has a significantly lower thermal conductivity even for very long nanowires.

(ii) While the thermal conductivity of a pure nanowire exhibits an inverse temperature dependence, that of a core-shell nanowire is practically temperature independent over a wide temperature range due to the diffusive nature of the majority of the vibrational modes present in core-shell nanowires.

(iii) The core-shell nanowire features a minimum and a crossover in the dependence of the thermal conductivity on cross section, as compared to the monotonic increase of a pure $\mathrm{Si}$ or $\mathrm{Ge}$ nanowire. The difference in behavior is attributed to changes in the relative magnitude of competing contributions to the heat conduction from thermal vibrations in the core center vs the shell surface, as core diameter changes.

Core-shell nanocomposite structures offer some control of phonon interference at the interface through manipulation of the interfacial interaction strength, which renders them promising for high-efficiency nanostructured thermoelectrics. Inspired by our simulation, further efforts should be focused on searching for a shell material with very strong interaction strength with the core, while the thermal conductivity of the outer shell is less important.

\section{ACKNOWLEDGMENTS}

K.P.G. expresses his gratitude to ETH Zurich for a visiting professorship grant. X.Z. gratefully acknowledges the scholarship of the State Scholarship Fund of the China Scholarship Council that allowed him to be a visiting scientist at ETH Zurich and contribute to this work. Computational support from the Brutus Cluster at ETH Zurich is gratefully acknowledged. This work was supported by a grant from the Swiss National Supercomputing Centre-CSCS under project ID s243. M.H. would like to thank Javier V. Goicochea for fruitful discussions. 
*Corresponding author: hum@ethz.ch

† giapis@cheme.caltech.edu

${ }^{\ddagger}$ dimos.poulikakos@ethz.ch

${ }^{1}$ G. Chen, M. S. Dresselhaus, G. Dresselhaus, J. P. Fleurial, and T. Caillat, Int. Mater. Rev. 48, 45 (2003).

${ }^{2}$ K. Hippalgaonkar, B. Huang, R. Chen, K. Sawyer, P. Ercius, and A. Majumdar, Nano Lett. 10, 4341 (2010).

${ }^{3}$ A. I. Hochbaum, R. Chen, R. D. Delgado, W. Liang, E. C. Garnett, M. Najarian, A. Majumdar, and P. Yang, Nature 451, 163 (2008).

${ }^{4}$ A. I. Boukai, Y. Bunimovich, J. Tahir-Kheli, J. K. Yu, W. A. Goddard, and J. R. Heath, Nature 451, 168 (2008).

${ }^{5}$ X. Chen, Y. Wang, Y. Ma, T. Cui, and G. Zou, J. Phys. Chem. C 113, 14001 (2009).

${ }^{6}$ D. Donadio and G. Galli, Phys. Rev. Lett. 102, 195901 (2009).

${ }^{7}$ J. Chen, G. Zhang, and B. Li, Nano Lett. 10, 3978 (2010).

${ }^{8}$ M. Hu, K. P. Giapis, J. V. Goicochea, X. Zhang, and D. Poulikakos, Nano Lett. 11, 618 (2011).

${ }^{9}$ X. Chen, Y. Wang, and Y. Ma, J. Phys. Chem. C 114, 9096 (2010).

${ }^{10}$ F. H. Stillinger and T. A. Weber, Phys. Rev. B 31, 5262 (1985).

${ }^{11}$ K. Ding and H. C. Anderson, Phys. Rev. B 34, 6987 (1986).

${ }^{12}$ M. Karimi, T. Kaplan, M. Mostoller, and D. E. Jesson, Phys. Rev. B 47, 9931 (1993).

${ }^{13}$ C. Roland and G. H. Gilmer, Phys. Rev. B 47, 16286 (1993).

${ }^{14}$ S. Plimpton, J. Comput. Phys. 117, 1 (1995).

${ }^{15}$ S. Nosé, J. Chem. Phys. 81, 511 (1984); W. G. Hoover, Phys. Rev. A 31, 1695 (1985).
${ }^{16}$ P. K. Schelling, S. R. Phillpot, and P. Keblinski, Phys. Rev. B 65, 144306 (2002).

${ }^{17}$ J. C. Li, C. L. Wang, H. Peng, M. X. Wang, R. Z. Zhang, H. C. Wang, J. Liu, M. L. Zhao, and L. M. Mei, J. Appl. Phys. 108, 063702 (2010).

${ }^{18}$ S. C. Wang, X. G. Liang, X. H. Xu, and T. Ohara, J. Appl. Phys. 105, 014316 (2009)

${ }^{19}$ X. Yang, A. C. To1, and R. Tian, Nanotechnology 21, 155704 (2010).

${ }^{20}$ N. Yang, G. Zhang, and B. W. Li, Nano Today 5, 85 (2010).

${ }^{21}$ C. J. Glassbrenner and G. A. Slack, Phys. Rev. 134, A1058 (1964).

${ }^{22}$ D. Li, Y. Wu, P. Kim, L. Shi, P. Yang, and A. Majumdar, Appl. Phys. Lett. 83, 2934 (2003).

${ }^{23}$ R. Chen, A. I. Hochbaum, P. Murphy, J. Moore, P. Yang, and A. Majumdar, Phys. Rev. Lett. 101, 105501 (2008).

${ }^{24}$ D. Donadio and G. Galli, Nano Lett. 10, 847 (2010).

${ }^{25}$ N. Mingo and D. A. Broido, Phys. Rev. Lett. 93, 246106 (2004).

${ }^{26}$ R. J. Bell and P. Dean, Discuss. Faraday Soc. 50, 55 (1970).

${ }^{27}$ J. L. Feldman and N. Bernstein, Phys. Rev. B 70, 235214 (2004).

${ }^{28}$ W. Lu, J. Xiang, B. P. Timko, Y. Wu, and C. M. Lieber, Proc. Natl. Acad. Sci. USA 102, 10046 (2005).

${ }^{29}$ J. Xiang, W. Lu, Y. Hu, Y. Wu, H. Yan, and C. M. Lieber, Nature 441, 489 (2006).

${ }^{30}$ E. S. Landry, Ph.D. thesis, Carnegie Mellon University, 2009.

${ }^{31}$ M. Posselt and A. Gabriel, Phys. Rev. B 80, 045202 (2009). 